\title{
Neutrophil response to cyclophosphamide predicts resil- ience to age-related learning impairment
}

\author{
Katie Nickel ${ }^{\mathrm{a}}$, Marianne Bjorner ${ }^{\mathrm{a}}$, Warren Ladiges ${ }^{\mathrm{a},{ }^{*},}$, Lida Zhu ${ }^{\mathrm{a}}$ \\ ${ }^{a}$ Department of Comparative Medicine, School of Medicine, University of Washington, Seattle, WA, USA.
}

\begin{abstract}
The ability to respond to stress, defined as resilience, was measured by white blood cell counts in C57BL/6 mice of various ages receiving a nonlethal dose of cyclophosphamide (CYP). Neutrophil counts dipped and then rebounded in a consistent and age-dependent manner. Low neutrophil rebound correlated with improved learning in middle-age mice suggesting CYP-nduced neutrophil response may predict resilience to aging.

Keywords: Resilience to aging, wound healing, ear punch biopsy, aging mice
\end{abstract}

Resilience is the ability to respond and recover from stressors which disrupt homeostasis [1]. Cyclophosphamide (CYP) is a chemotherapeutic drug that halts cell cycling of precursor neutrophils, which decreases the number of mature neutrophils until the drug is eliminated from body [2]. In the model described in this report, CYP was used as a stressor, where the resilience of mice to respond to one dose was quantified by changes in circulating neutrophil percentages and compared to the aging parameter of cognitive impairment.

C57Bl/6 male mice aged 4, 12, 17, and 28 months old were injected with $100 \mathrm{mg} / \mathrm{kg}$ CYP intraperitoneally. Control groups were injected with $0.9 \%$ saline. A drop of tail blood was collected periodically after injection and white blood cells were counted (Figure 1). A spatial navigation task (Box Maze) was used to assess learning ability [3] (Figure 2). Cohorts of 8-10 mice per age group were used.

The neutrophil percentages followed a predictable pattern across age groups: an initial increase after injection, then a decrease reaching a nadir at day 5 , and a rebound at day 7 (Figures 3A, 3B, 3C, and 3D). The degree to which neutrophil percentages changed was dependent upon age; in particular, the strength of the neutrophil percentage rebound from day 5 to day 7 decreased with increasing age (Figure 4A). Neutrophil rebound negatively correlated with escape speed from a box maze, a learning paradigm

\footnotetext{
* Corresponding author: Warren Ladiges

Mailing address: Department of Comparative Medicine, School of Medicine, University of Washington, Seattle, WA, USA.

Email: wladiges@uw.edu
}

Received: 30 November 2020 / Accepted: 15 December 2020

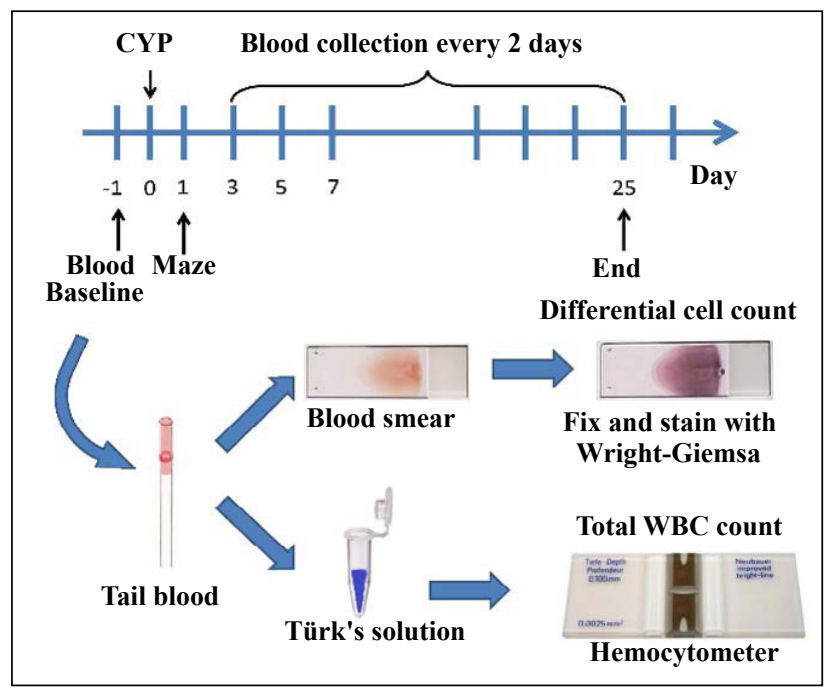

Figure 1. Timeline of experiment and description of the white blood cell counting procedure.

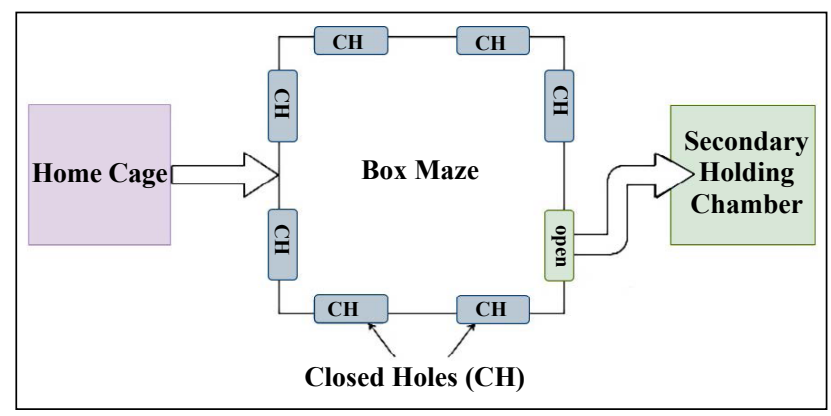

Figure 2. Spatial navigation task designated as the Box maze. Five 2-minute trials were conducted per mouse, noting time to reach an open escape hole. 


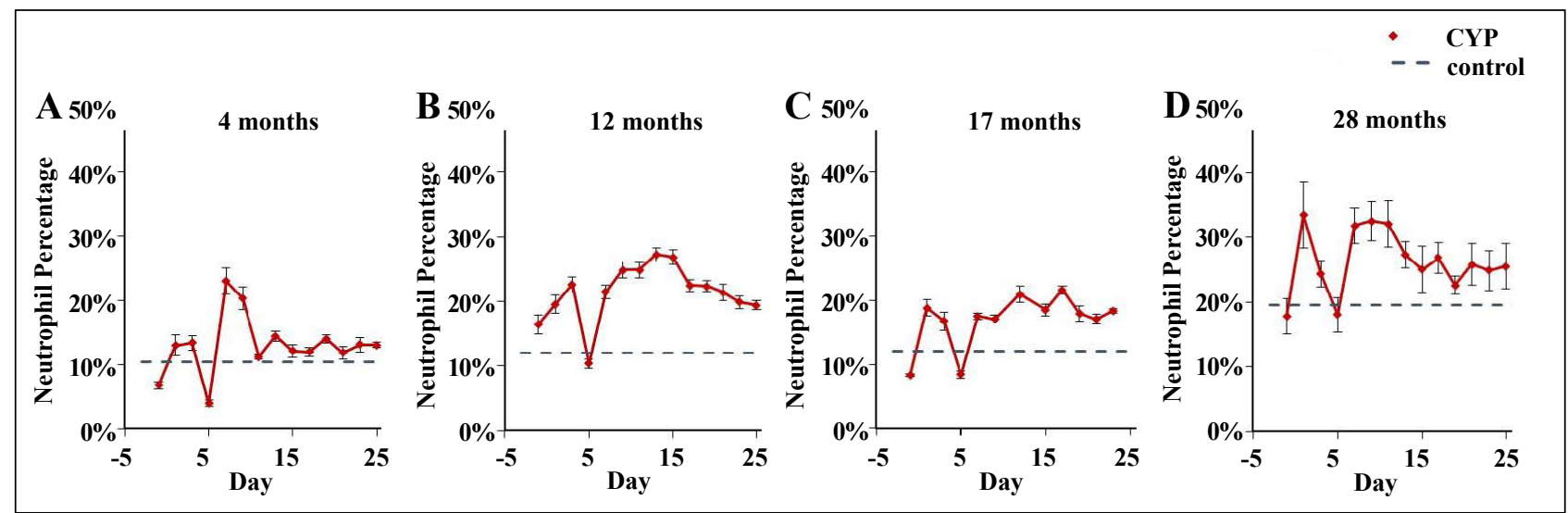

Figure 3. Change in neutrophil percentage relative to total white blood cell counts for both control and CYP injected mice over a period of 25 days. Mice were separated by age at (A) 4 months; (B) 12 months; (C) 17 months; and (D) 27 months.

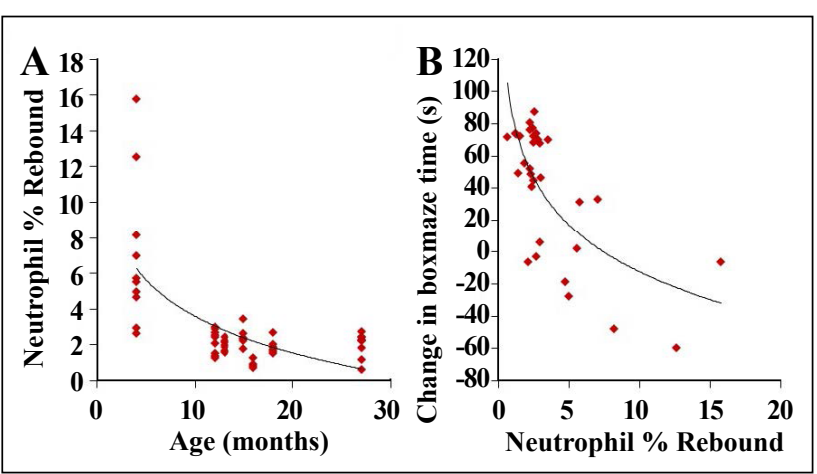

Figure 4. (A) As the age of mice increased, the strength of the neutrophil rebound decreased. (B) The change in escape times in the spatial navigation task (Box maze) from trial 1 to trial 5 was negatively correlated with the neutrophil rebound response.

where higher speeds are associated with resilience to agerelated cognitive decline (Figure 4B).

In summary, one non-lethal dose of CYP induced a neutrophil rebound response that was consistent over all age groups as previously reported [4]. The rebound strength decreased with increasing age, so it was age-dependent. A low CYP-induced neutrophil rebound response was associated with decreased learning impairment. This may predict resilience to aging given the correlation with established cognitive aging parameters, though the cause of this relationship requires further study. These observations provide a preclinical model for developing clinical in vitro investigations using CYP-induced stress in human peripheral white blood cells.

\section{Declarations}

Acknowledgments: This work was supported by NIA grant R01 AG057381 (Ladiges, PI).

Conflict of Interest: Warren Ladiges is a member of the Editorial Board of Aging Pathobiology and Therapeutics. All authors declare no conflict of interest and were not involved in the journal's review or desicions related to this manuscript.

\section{References}

1. Schorr A, Carter C, Ladiges W. The potential use of physical resilience to predict healthy aging. Pathobiology of Aging \& Age-related Diseases, 2018, 8(1): 1403844.

2. Emmenegger U, Man S, Shaked Y, et al. A comparative analysis of low-dose metronomic cyclophosphamide reveals absent or low-grade toxicity on tissues highly sensitive to the toxic effects of maximum tolerated dose regimens. Cancer Research, 2004, 64(11): 3994-4000.

3. Darvas M, Mukherjee K, Lee A, et al. A Novel One-Day Learning Procedure for Mice. Current Protocols in Mouse Biology, 2020, 10(1): e68.

4. Zhu L, Dou Y, Bjorner M, et al. Development of a cyclophosphamide stress test to predict resilience to aging in mice. GeroScience, 2020: 1-9.

Cite this article as: Katie N, Marianne B, Warren L, et al. Neutrophil response to cyclophosphamide predicts resilience to age-related learning impairment[J]. Aging Pathobiology and Therapeutics, 2020, 2(4): 230-231. 